\title{
Le Programme international pour le suivi des acquis des élèves (PISA)
}

The Programme for International Student Assessment (PISA)

El programa internacional para el seguimiento de los Conocimientos de los

Alumnos (PISA)

Jacqueline Levasseur

\section{OpenEdition}

\section{Journals}

Édition électronique

URL : http://journals.openedition.org/ries/257

DOI : 10.4000 /ries.257

ISSN : 2261-4265

\section{Éditeur}

Centre international d'études pédagogiques

\section{Édition imprimée}

Date de publication : 1 décembre 2006

Pagination : 127-140

ISSN : 1254-4590

\section{Référence électronique}

Jacqueline Levasseur, "Le Programme international pour le suivi des acquis des élèves (PISA)»,

Revue internationale d'éducation de Sèvres [En ligne], 43 | décembre 2006, mis en ligne le 05 avril 2012, consulté le 30 avril 2019. URL : http://journals.openedition.org/ries/257 ; DOI : 10.4000/ries.257 


\section{Le Programme international pour le suivi des acquis des élèves (PISA)}

\section{Jacqueline Levasseur}

Dans quelle mesure les jeunes adultes sont-ils prêts à affronter les défis de demain? Répondre à cette question est le but essentiel du programme PISA de l'OCDE. Depuis près de quinze ans, l'OCDE produit et publie des indicateurs chiffrés permettant de comparer les systèmes éducatifs de ses pays membres : les moyens consacrés à l'enseignement (les structures, les enseignants, le financement...), les effectifs, les diplômés, les résultats de l'éducation en lien avec le marché du travail... Ces indicateurs comparent des caractéristiques des systèmes éducatifs mais ne donnent pas d'informations sur les acquis des élèves. C'est pour combler cette lacune que les gouvernements des pays membres ont souhaité se doter d'un vaste programme d'évaluation des acquis des élèves.

Dès sa conception, le programme PISA a été défini pour permettre aux pays membres de l'OCDE de recueillir par une enquête internationale commune, de manière cyclique, tous les trois ans, des informations sur les compétences acquises par les élèves arrivant en fin de scolarité obligatoire.

Le programme PISA de l'OCDE n'est pas la première enquête internationale comparative sur les acquis des élèves. De nombreuses études ont été menées au cours des cinquante dernières années. Les plus largement connues sont celles conduites par l'association internationale pour l'évaluation du rendement scolaire (IEA), et celles réalisées dans le cadre de l'International Assessment of Educational Progress (IAEP) par Education Testing Service (ETS). Ces enquêtes sont principalement centrées sur les acquisitions des élèves en lien direct avec les programmes d'enseignements disciplinaires, et ont donc porté sur les parties de programmes - en principe - communes aux pays participants. Par rapport à ces enquêtes, le PISA est inédit à bien des égards.

\section{UNE INITIATIVE DES PAYS MEMBRES DE L'OCDE}

Le Programme international pour le suivi des acquis des élèves (PISA) a été créé en 1997 pour compléter l'ensemble des indicateurs des systèmes d'enseignement de l'OCDE (programme INES). Ces indicateurs quantitatifs, comparables à l'échelle internationale, publiés chaque année, n’intégraient pas 
d'indicateurs d'acquis des élèves. Cette décision exprimait la volonté des gouvernements des pays membres d'étudier de façon régulière, à l'intérieur d'un cadre conceptuel commun, les résultats des systèmes éducatifs nationaux en termes d'acquis des élèves. Ce programme est le fruit d'un effort concerté entre les pays qui veulent mettre en œuvre des enquêtes régulières pour le pilotage de leur politique éducative.

Les pays participants ${ }^{1}$ assument la responsabilité politique du projet. L'organisation et la structure de pilotage du projet permettent à l'OCDE et aux pays participants de veiller à la qualité et à la validité des instruments d'évaluation : de façon à permettre une comparaison internationale, ces instruments sont identiques pour tous les élèves des pays différents; ils doivent également prendre en compte le contexte culturel et éducatif des pays différents, avoir des qualités psychométriques robustes, permettre des comparaisons tant géographiques que temporelles, mettre en relation des acquis d'élèves et des contextes.

\section{L'objectif général de PISA}

PISA vise à déterminer dans quelle mesure les élèves de quinze ans sont préparés à relever les défis des sociétés dans lesquelles ils vivront demain. L'approche retenue est une évaluation portant sur des connaissances et des compétences qui, au-delà des programmes d'enseignements disciplinaires, reflètent la capacité des jeunes à utiliser leurs acquis dans des situations de la vie courante. Les compétences évaluées doivent rendre compte de la capacité des jeunes à appliquer ce qu'ils ont appris, à poursuivre leur formation tout au long de la vie, à prendre des décisions.

Pour répondre à cet objectif, PISA combine l'évaluation dans trois domaines principaux : la compréhension de l'écrit, la culture mathématique, la culture scientifique. Le PISA réalise une enquête tous les trois ans qui vise plus largement l'un des trois domaines. Ainsi, pour le premier cycle, 2000 à 2006, l'année 2000 concernait en majeure la compréhension de l'écrit, l'année 2003 couvrait majoritairement la culture mathématique, la majeure de l'année 2006 était la culture scientifique. Un second cycle reprend en 2009 avec à nouveau la compréhension de l'écrit en domaine majeur. Les évaluations cognitives des élèves sont complétées par des informations sur les contextes socio-éducatifs.

\section{Le groupe d'âge retenu}

La population ciblée est celle des élèves de quinze ans et non pas les élèves inscrits dans un niveau scolaire donné. Ce choix est nouveau dans le champ des enquêtes internationales. Par exemple, les enquêtes TIMSS ${ }^{2}$ menées

\footnotetext{
1. En 2000,28 pays membres de l'OCDE et 4 pays externes; en 2003 les 30 pays membres actuels de l'OCDE et 11 externes; en 2006 les 30 pays membres de l'OCDE et 26 pays non-membres.

2. Trends in International Mathematics and Science Study.
} 
par l'IEA ou Reading litteracy ${ }^{3}$ réalisées dans le cadre de l'IAEP s'intéressent aux élèves dans des niveaux scolaires précis et ciblent les programmes relatifs à ces niveaux. Pour la première fois, avec PISA, l'évaluation porte sur une cohorte d'élèves, les élèves de quinze ans, quel que soit leur niveau de scolarisation.

Dans la plupart des pays de l'OCDE, l'âge de quinze ans est celui de la fin de la scolarité obligatoire (ou de base) dans le cadre de laquelle tous les élèves ont suivi un programme d'enseignement pratiquement commun. Cependant, la plupart des jeunes des pays de l'OCDE poursuivent leurs études au-delà de quinze ans. Évaluer les jeunes en fin de scolarité obligatoire fournit des indications très utiles sur la performance des systèmes éducatifs : il est important de déterminer dans quelle mesure les élèves ont acquis, à ce stade, les connaissances et compétences qui leur serviront ultérieurement, y compris dans leurs parcours de formation. Ce choix politique rend très hasardeuses les comparaisons avec les enquêtes existantes telles que TIMSS, mais il est meilleur pour la comparabilité des résultats dans le contexte du PISA. En effet, les concepteurs ont supposé que, quel que soit le pays, les jeunes de quinze ans seront confrontés à des situations similaires nécessitant la mobilisation de leurs compétences, a priori indépendantes des différents contextes culturels.

Toutefois, il faut préciser qu'il ne s'agit pas, dans tous les pays, de toute la génération des élèves de quinze ans. La population définitivement ciblée dans le PISA est celle de jeunes de quinze ans inscrits dans un établissement scolaire, ce qui représente un pourcentage très variable des élèves de quinze ans : $96 \%$ au Royaume-Uni, $95 \%$ en Autriche, $93 \%$ en Australie, $85 \%$ en Corée, près de $100 \%$ en France, contre environ $60 \%$ seulement au Mexique et en Turquie.

\section{Le choix des contenus}

Les connaissances et compétences retenues dans les évaluations pour PISA ne se limitent pas à un dénominateur commun des programmes d'enseignements disciplinaires nationaux. Il s'agit là de la caractéristique la plus importante et la plus ambitieuse du programme PISA. Ce choix différencie fondamentalement le PISA des autres enquêtes internationales conduites en milieu scolaire.

Traditionnellement, les programmes scolaires sont surtout conçus en tant qu'ensembles de connaissances et de techniques à acquérir et accordent une moindre importance aux compétences qui devraient être développées dans la perspective plus générale d'une application dans les situations rencontrées dans la vie courante. Les choix pour le programme PISA se situent dans l'évaluation de la maîtrise de compétences permettant de mobiliser et d'exploiter les connaissances acquises dans le contexte des programmes d'enseignement. Le PISA se

3. Compréhension de l'écrit. 
définit comme une évaluation de compétences assez générales, qui ne dépendent pas d'un enseignement spécifique et prend tout son sens en référence à la volonté des pays de développer le capital humain : "les connaissances, les savoir-faire, les compétences et autres caractéristiques des individus qui sont pertinentes pour le bien-être personnel, social et économique».

Du fait qu'il évalue les connaissances et les compétences maîtrisées à un moment proche de la fin de la scolarité obligatoire, le PISA informe sur l'état de préparation des jeunes de quinze ans à la vie adulte, et dans une certaine mesure, sur l'efficacité des systèmes éducatifs. Son objectif est d'évaluer les performances des élèves par rapport aux objectifs fondamentaux des systèmes éducatifs, définis par la société, et non par référence aux corpus de connaissances techniques désignés comme objets d'apprentissage dans les cursus scolaires. Cette approche incite alors les systèmes éducatifs à s'intéresser aux défis posés par les sociétés contemporaines.

\section{Les grands types d'indicateurs}

Le programme PISA est conçu pour fournir trois grands types d'indicateurs :

- des «indicateurs de base» qui cernent le profil général des compétences des élèves dans les domaines évalués;

- des «indicateurs contextuels» qui montrent en quoi ces compétences sont liées à différentes variables démographiques, sociales, économiques, éducatives décrivant les élèves, les établissements et les systèmes éducatifs ;

- des «indicateurs de série temporelle» qui, grâce à la périodicité du recueil des informations, montrent l'évolution des niveaux de compétences, l'évolution de leurs liens avec les contextes.

Ces indicateurs sont un moyen d'attirer l'attention sur des problématiques importantes, mais ne répondent pas directement aux questions que se posent les décideurs politiques. Ils doivent, au-delà d'une simple présentation, être analysés au regard des choix de politiques éducatives propre à chaque pays.

\section{Les méthodes d'élaboration}

L'élaboration progressive, depuis 1997, du programme PISA est le résultat des efforts concertés des pays membres de l'OCDE. Les enquêtes successives sont élaborées et approuvées par les pays participants qui assurent ensuite leur mise en œuvre.

Le conseil des pays participants (PGB) est la réunion des représentants officiels de chaque pays; il définit les objectifs et veille au respect de ces objectifs tout au long du processus de mise en œuvre. Il détermine les objectifs concernant la mise au point des instruments d'évaluation et des questionnaires de contextes, l'élaboration des indicateurs, la présentation des résultats. 
Le secrétariat de l'OCDE est responsable de la gestion globale du PISA. Il suit sa mise en œuvre, il produit les indicateurs internationaux et les analyses, il publie les rapports internationaux.

Des experts scientifiquement reconnus, de nombreux pays participants, collaborent dans les différents groupes de travail. Ces groupes d'experts veillent à la validité internationale des instruments et à la qualité des procédures.

La conception et mise en œuvre des enquêtes sont confiées à un consortium international, qui était, pour le cycle 2000 à 2006, dirigé par l'Australian Council for Educational Research (ACER) en Australie. Les membres de ce consortium étaient outre ACER, CITO (Institut national pour l'évaluation de l'enseignement) aux Pays-Bas, WESTAT et ETS aux États-Unis, NIER (National Institute for Educational Policy Research) au Japon.

Les pays participants développent alors le programme PISA à l'échelon national par l'intermédiaire des «centres nationaux» et des «chefs de projets nationaux (NPM)».

Au cours des neuf dernières années, le travail collectif a, entre autres, conduit à élaborer et à valider un cadre conceptuel pour chacun des domaines d'évaluation (compréhension de l'écrit, culture mathématique, culture scientifique), à améliorer la qualité des mesures, à identifier des caractéristiques clés à prendre en considération pour construire des épreuves internationales.

Chaque enquête est construite selon le même schéma : des épreuves standardisées "papier-crayon» développées au niveau international, d'une durée de deux heures par élève; les épreuves comprennent des items à choix multiple et des questions ouvertes demandant aux élèves de construire leur réponse; le principe des «cahiers tournants» permet d'évaluer les élèves dans un maximum de compétences; l'ensemble des épreuves par domaine représente au moins sept heures d'évaluation, mais chaque élève ne répond qu'à une partie sur la durée de deux heures; un questionnaire contextuel par élève (durée de trente minutes) contient des questions sur eux-mêmes, leur contexte socio-familial, leurs motivations, goûts, aspirations; un questionnaire contextuel par chef d'établissement (vingt minutes) porte sur des caractéristiques de l'établissement.

L'implication des gouvernements s'est traduite par un souci de rigueur méthodologique pour éviter une remise en cause des résultats. Des méthodologies particulières sont utilisées pour le tirage des échantillons et leur vérification, pour la traduction des épreuves, pour le codage des réponses des élèves.

Un contrôle de la qualité du suivi des procédures est mis en place par le consortium international et l'OCDE à tous les niveaux : les centres nationaux et les NPM, les établissements de l'échantillon et l'administration des épreuves, les correcteurs par un système de double et quadruple correction, la saisie et le nettoyage des données, la constitution des fichiers. 


\section{Ce Qui est Évalué DANS CHAQUE DOMAINE DU PISA}

Chaque année d'enquête fait porter l'évaluation sur l'un des trois domaines considérés alors comme domaine "majeur" auquel sont consacrés les deux tiers de la durée des épreuves; les deux autres domaines sont dits «mineurs».

\section{Définition de chaque domaine}

Un trait essentiel de la définition des domaines dans PISA est le recours au concept de «culture» ou «littératie» pour mettre l'accent sur les connaissances et les compétences nécessaires pour être efficace dans la vie quotidienne.

Dans la vie quotidienne, la compréhension de l'écrit exige bien davantage que savoir décoder de l'écrit, interpréter le sens des mots et des structures grammaticales, construire du sens. Elle dépend aussi de la capacité à réfléchir sur le sens d'un texte, à saisir l'implicite d'un texte, à identifier les procédés de l'auteur pour faire comprendre son message et influencer le lecteur, faire des inférences de sens à partir de la structure et des caractéristiques du texte. La compréhension de l'écrit est aussi la capacité à comprendre et interpréter des

132 textes de type très divers, de construire du sens à partir de plusieurs documents écrits en les reliant au contexte dans lequel ils sont proposés.

De même, la culture mathématique exige plus que de maîtriser les propriétés des nombres et des opérations, les notions d'espace et de forme, les notions de mesure, celles de variations, de croissance et d'incertitudes, etc. La culture mathématique, c'est aussi la capacité à penser et à procéder de «façon mathématique », à résoudre des problèmes, à comprendre et apprécier des arguments mathématiques, à poser des problèmes, à savoir s'exprimer sur des sujets à contenu mathématique.

La culture scientifique, liée elle aussi à un corpus de connaissances telles que, par exemple, les concepts fondamentaux de respiration, de circulation sanguine, de chaînes alimentaires, des états de la matière, de taux de réaction, etc. La culture scientifique repose également sur la capacité à mettre en œuvre des processus d'investigation scientifique, par exemple, reconnaître les limites d'une investigation, être capable d'identifier des faits pertinents pour répondre à une question ou encore évaluer et communiquer des conclusions.

Les concepteurs du programme PISA ont ainsi adopté des définitions communes de la compréhension de l'écrit, de la culture mathématique, de la culture scientifique (voir encadré 1). Dans chacun de ces domaines, l'accent est mis sur la capacité de réfléchir et d'utiliser des modes de raisonnement, d'utiliser des connaissances et des compétences liées à la lecture, aux mathématiques et aux sciences (physique, chimie, sciences de la vie et de la terre). 


\section{Définitions OCDE/PISA de la compréhension de l'écrit, de la culture mathématique et scientifique}

\section{Compréhension de l'écrit}

C'est non seulement utiliser des textes écrits, mais aussi réfléchir à leur propos, les analyser. Cette capacité doit permettre à chacun de pouvoir réaliser ses objectifs, développer ses connaissances et son potentiel et jouer un rôle actif dans la société.

\section{Culture mathématique}

C'est pouvoir identifier et comprendre les divers rôles joués par les mathématiques dans le monde, porter des jugements fondés à leurs propos, et s'engager dans des activités mathématiques, en fonction des exigences que rencontre l'individu dans sa vie privée, sociale et professionnelle tant présente que future, en tant que citoyen constructif, impliqué et réfléchi.

\section{Culture scientifique}

C'est pouvoir utiliser des connaissances scientifiques pour être capable d'identifier les questions auxquelles la recherche scientifique peut apporter des réponses et pour tirer des conclusions fondées sur des faits, en vue de comprendre le monde naturel et les changements qui y sont apportés par l'activité humaine, et de contribuer à prendre des décisions à ce propos.

Source : OCDE 2000 et 2003.

\section{Les trois dimensions structurant chaque domaine}

Ces définitions ont permis d'articuler l'évaluation dans chaque domaine selon trois dimensions : le type de tâche ou processus, les contenus, l'usage ou le contexte d'application.

\section{Les types de tâches (ou processus)}

La compréhension de l'écrit, la culture mathématique et la culture scientifique, sollicitent chacune un grand nombre de types de tâches qui interviennent

\section{Les types de tâches dans les trois domaines d’OCDE/PISA}

\section{Compréhension de l'écrit}

«Exécution de diverses tâches de lecture telles que la compréhension générale d'ensemble, trouver des informations, développer une interprétation, réfléchir sur le contenu ou la forme du texte».

\section{Culture mathématique}

«Application de compétences mathématiques (par exemple : modélisation, résolution de problèmes), classées en trois catégories : 1) exécuter des procédures; 2 ) mettre en relation et résoudre des problèmes; 3) "mathématiser", c'est-à-dire opérer une traduction de la réalité vers la structure mathématique), effectuer des raisonnements mathématiques et généraliser».

\section{Culture scientifique}

«Mise en œuvre de démarches scientifiques - par exemple reconnaitre les questions qui peuvent être résolues de manière scientifique, identifier des faits, tirer des conclusions, les évaluer, les communiquer et montrer une compréhension globale des concepts scientifiques. Ces démarches n'impliquent pas nécessairement un ensemble de connaissances scientifiques préalables mais doivent nécessairement être mises en œuvre en relation avec des contenus scientifiques». 
dans la détermination du niveau de compétences de chaque individu. Les types de tâches sont déterminés par les capacités cognitives mises en jeu et les caractéristiques des questions posées.

\section{Les contenus}

Le programme PISA retient, dans les questions des épreuves, les savoirs et savoir-faire transmis par les programmes d'enseignement, mais il n'évalue pas leur maîtrise en tant que telle car il ne s'agit pas, avec PISA, d'une évaluation des performances scolaires. Au fur et à mesure de son développement, chaque individu améliore son niveau de compétences et augmente son capital de connaissances et de savoir-faire. Le champ, dans chaque domaine, est alors si vaste qu'il ne peut être exhaustivement couvert dans une évaluation. Aussi, les concepteurs de PISA ont choisi d'évaluer les compétences en se référant à des savoirs et savoir-faire généraux qui permettent l'application des savoirs «scolaires» progressivement acquis.

\section{Les contenus dans les trois domaines d’OCDE/PISA}

\section{Compréhension de l'écrit}

Lecture de différents types de textes : textes continus, divisés en sous-catégories (par exemple : textes descriptifs, narratifs, informatifs, argumentatifs ou instructifs) et documents classés selon leur structure (par exemple : formulaires, annonces, publicités, graphiques ou tableaux).

\section{Culture mathématique}

Pour l'essentiel, les «idées mathématiques majeures» utilisées dans PISA sont : les variations et les relations de dépendance, l'espace et les formes, le raisonnement quantitatif, les probabilités et les statistiques.

\section{Culture scientifique}

Concepts scientifiques : par exemple structure et propriétés de la matière, modifications chimiques et physiques, transformation d'énergie, forces et mouvements, formes et fonctions, biologie humaine, biodiversité, contrôle génétique, se rapportant aux domaines majeurs de la science (physique, biologie, chimie, etc.) et utilisés en vue de résoudre des problèmes touchant aux sciences de la vie et à la santé, aux sciences appliquées à la Terre et à l'environnement, et aux domaines technologiques.

Source : OCDE 2000.

\section{Les situations}

Dans la vie quotidienne, les situations d'application et d'utilisation de connaissances et compétences en compréhension de l'écrit sont multiples, de même pour la culture mathématique et la culture scientifique. Les définitions dans PISA incluent que l'individu est capable de mettre en ouvre et d'exploiter ses connaissances et savoir-faire dans une gamme très étendue de situations. L'encadré suivant présente, par domaine, l'esprit général retenu dans le choix des situations pour l'évaluation des compétences des élèves de quinze ans. 


\section{Les situations dans les trois domaines d'OCDE/PISA}

\section{Compréhension de l'écrit}

«Lire des textes écrits en vue d'utilisations différentes, par exemple, pour son intérêt personnel ou à des fins professionnelles».

\section{Culture mathématique}

«Utiliser les mathématiques dans diverses situations, par exemple, pour traiter des problèmes qui concernent l'individu dans un contexte personnel, éducatif ou public».

\section{Culture scientifique}

«Utiliser les sciences dans diverses situations, par exemple pour traiter des problèmes qui concernent un individu, une communauté ou le monde entier».

Source : OCDE 2000.

\section{L'apport des questionnaires de contexte}

Ces questionnaires aux élèves et aux chefs d'établissements recueillent notamment des informations sur:

- la biographie de l'élève, le contexte social et culturel dans lequel il évolue;

- les attitudes de l'élève à l'égard de sa formation, ses habitudes et méthodes de travail dans le milieu scolaire et dans le milieu familial;

- les ressources matérielles et humaines de l'établissement, la gestion, les pratiques de participation à la décision dans l'établissement;

- les structures de l'établissement;

- les stratégies éducatives, les relations entre/avec les enseignants, les relations entre/avec les élèves, avec les parents.

Par ailleurs, un questionnaire sur la familiarité des élèves avec les technologies de l'information a été proposé en option.

Ces informations contextuelles permettent de compléter l'ensemble des données dont dispose l'OCDE dans le cadre des indicateurs descriptifs des systèmes éducatifs (INES).

L'élaboration de ce cadre conceptuel a bénéficié de l'apport scientifique d'experts de nombreux pays membres de l'OCDE. Ce cadre est l'ossature des enquêtes réalisées en 2000, 2003 et 2006. Il constituera la base du cadre conceptuel du prochain cycle PISA dont la première prise d'informations est prévue en 2009 avec pour domaine majeur la compréhension de l'écrit.

\section{Que nous apprend le PISA?}

Les informations rassemblées dans le cadre du programme PISA permettent, au-delà de comparaisons aboutissant à un palmarès des pays, d'étudier les résultats en analysant les facteurs de contexte associés à la performance des élèves. 
Ce sont les indicateurs de base, ceux qui décrivent hors contexte la maîtrise des compétences des élèves de quinze ans qui ont été le plus exploités par les médias en ne retenant que les aspects de palmarès, alors que les écarts des performances entre pays peuvent être étudiés au regard des différentes variables contextuelles liées à l'élève et à son milieu socio-culturel, à l'établissement.

Le but premier du PISA consiste à comparer les acquis des jeunes de quinze ans des pays participants en tenant compte des spécificités propres à chaque système éducatif. Les indicateurs comparables au plan international permettent d'établir les niveaux de compétences atteints dans les différents pays.

\section{Un descriptif des compétences maîtrisées}

La dimension "types de tâches» est, pour chaque domaine, mesurée sur des échelles de compétences ou de contenus : trois échelles en 2000 pour la compréhension de l'écrit, quatre échelles en 2003 pour la culture mathématique, une échelle en 2000 et 2003 pour la culture scientifique. Dans chaque domaine, les échelles sont indépendantes et il n'y a pas de hiérarchie entre elles.

Pour la compréhension de l'écrit, l'échelle "s'informer» renvoie aux compétences des élèves à localiser des informations dans un contexte, l'échelle « interpréter» correspond aux compétences des élèves à dégager du sens et à établir des inférences, l'échelle "réagir » aux compétences des élèves, à mettre en relation le texte avec leurs connaissances, leurs idées et leurs expériences. Une échelle globale de compréhension de l'écrit combine les compétences de ces trois échelles.

De la même manière, pour la culture mathématique, une échelle synthétique de "culture mathématique» se décompose en quatre échelles : " espace et forme», "variations et relations», «quantités», «incertitude».

La culture scientifique, majeure en 2006, devrait faire l'objet d'une description des compétences dans plusieurs échelles plus précises que l'échelle globale analysée en 2000 et 2003 (la culture scientifique était une mineure dans ces enquêtes).

Toutes les échelles sont construites selon le même modèle psychométrique de calcul des scores (modèle de réponse à l'item). Chaque échelle permet de classer les pays sur une dimension commune.

Sur chaque échelle, des seuils ont été définis afin de partager les élèves en "groupes de performances» correspondant à un profil de compétence. L'encadré suivant présente, pour le PISA 2000 en compréhension de l'écrit, la définition des groupes de performances et le profil général de compétences des élèves. Pour chaque pays, la répartition des élèves selon ces groupes permet de rendre compte de la dispersion des résultats de ce pays. 
Définition des groupes de performances en compréhension de l'écrit

\begin{tabular}{|c|c|c|}
\hline $\begin{array}{l}\text { Groupes de } \\
\text { performances }\end{array}$ & Seuils de score & Profil général des élèves \\
\hline Groupe 5 & $\begin{array}{l}\text { Score supérieur } \\
\text { à } 625\end{array}$ & $\begin{array}{l}\text { Les élèves sont capables de mener à bien des tâches } \\
\text { de lecture complexes, notamment de traiter } \\
\text { des informations difficiles à chercher dans des textes } \\
\text { qui ne leur sont pas familiers, de comprendre de manière } \\
\text { approfondie des textes de ce type et d'en dégager } \\
\text { les informations pertinentes pour la tâche à accomplir, } \\
\text { de procéder à des évaluations critiques et d'élaborer des } \\
\text { hypothèses, de faire appel à des connaissances spécialisées } \\
\text { et de recourir à des notions qui peuvent être inattendues. }\end{array}$ \\
\hline Groupe 4 & $\begin{array}{l}\text { Score compris } \\
\text { entre } 553 \text { et } 625\end{array}$ & $\begin{array}{l}\text { Les élèves sont capables d'effectuer des tâches difficiles } \\
\text { de lecture, notamment de localiser des informations } \\
\text { enfouies dans un texte, de dégager du sens à partir } \\
\text { des nuances de langage et d'évaluer un texte de manière } \\
\text { critique. }\end{array}$ \\
\hline Groupe 3 & $\begin{array}{l}\text { Score compris } \\
\text { entre } 481 \text { et } 552\end{array}$ & $\begin{array}{l}\text { Les élèves sont capables d'effectuer des tâches de lecture } \\
\text { d'une complexité modérée, notamment de localiser } \\
\text { de multiples fragments d'information, d'établir des liens } \\
\text { entre différentes parties de texte et de mettre l'écrit } \\
\text { en relation avec des connaissances familières. }\end{array}$ \\
\hline Groupe 2 & $\begin{array}{l}\text { Score compris } \\
\text { entre } 408 \text { et } 480\end{array}$ & $\begin{array}{l}\text { Les élèves sont capables d'effectuer des tâches } \\
\text { de lecture élémentaires, notamment de localiser des } \\
\text { informations directes, d'établir divers types d'inférences } \\
\text { d'un degré limité, de découvrir le sens d'un passage bien } \\
\text { défini d'un texte et d'utiliser des connaissances différentes } \\
\text { pour le comprendre. }\end{array}$ \\
\hline Groupe 1 & $\begin{array}{l}\text { Score compris } \\
\text { entre } 335 \text { et } 407\end{array}$ & $\begin{array}{l}\text { Les élèves de ce groupe sont uniquement capables } \\
\text { d'effectuer les tâches de lecture les moins complexes } \\
\text { de PISA, notamment de localiser un fragment unique } \\
\text { d'information, d'identifier le thème principal d'un texte } \\
\text { ou d'établir une relation simple avec des connaissances } \\
\text { de la vie courante. }\end{array}$ \\
\hline Groupe 0 & $\begin{array}{l}\text { Score inférieur } \\
\text { à } 335\end{array}$ & $\begin{array}{l}\text { Ces élèves ne sont pas capables de mettre couramment } \\
\text { en ouvre les connaissances et les compétences } \\
\text { les plus élémentaires que PISA cherche à mesurer. } \\
\text { Cela ne doit pas être interprété comme une absence } \\
\text { de compétences en lecture chez l'élève. En fait, la plupart } \\
\text { des élèves concernés sont vraisemblablement capables } \\
\text { de lire dans l'acception technique du terme mais éprouvent } \\
\text { de sérieuses difficultés à utiliser la lecture comme un outil } \\
\text { pour étendre et améliorer leurs connaissances } \\
\text { et leurs compétences dans d'autres domaines. }\end{array}$ \\
\hline
\end{tabular}




\section{Des éclairages apportés par les contextes}

Les réponses apportées par les élèves et les chefs d'établissement aux questionnaires donnent des informations très riches et variées, par exemple sur les attitudes des élèves envers leur formation, les caractéristiques du milieu scolaire etc. et permettent de construire des indices. Ceux-ci, croisés avec les performances, apportent des informations pour chaque pays sur les relations de ces variables avec les performances des élèves. Ces analyses fournissent aux pays un regard qualitatif sur leur système éducatif et montrent que ces relations ne sont pas équivalentes dans tous les pays. Ainsi, par exemple, en compréhension de l'écrit, il ressort que le milieu socio-économique a une importance plus forte sur la performance en France que pour la moyenne des pays de l'OCDE.

L'analyse qualitative des effets des contextes fait apparaître le rôle des systèmes, le poids des structures (filières, orientation...) et invite les pays à un regard sur leurs choix de politique éducative.

\section{QUE FONT LES PAYS AVEC LES DONNÉES DU PISA?}

Le programme PISA fournit aux pays une comparaison internationale des performances et des compétences de leurs élèves de quinze ans. Il donne ainsi des éléments sur l'efficacité des systèmes éducatifs. En France, par exemple, les résultats du PISA viennent en complément des enquêtes menées au niveau national. Il permet aux décideurs d'infirmer ou de confirmer des hypothèses concernant la qualité de leur propre système éducatif. Il permet aux pays de confronter leur perception de leur système éducatif à un ensemble extérieur.

Dans certains pays, le PISA semble constituer un objectif à atteindre (Hong-Kong, Corée) pour se maintenir en haut du classement des pays de l'OCDE. Dans d'autres, ce sont les comparaisons régionales des résultats obtenus au PISA qui priment pour les décideurs. Elles donnent un éclairage sur les orientations que le système éducatif pourrait prendre (Italie, Espagne).

Plus nombreux sont les pays qui prennent des références dans le PISA pour engager des réflexions de fond sur les finalités de leur système éducatif et introduire des réformes : ainsi la Hongrie et surtout l'Allemagne qui développe des standards communs aux Länder pour le pilotage de la formation et l'évaluation des établissements scolaires. Dans le même esprit, en France, le décret relatif au socle commun de connaissances et de compétences, fait explicitement référence au programme PISA.

On voit bien que l'enquête PISA, initialement mise en œuvre par les gouvernements des pays de l'OCDE pour répondre à leurs besoins spécifiques, est devenue au fil du temps un instrument politique majeur pour de nombreux autres pays et pour leur économie. Elle joue un rôle de plus en plus important 
dans diverses parties du monde : l'enquête a été ou est en voie d'être menée dans des pays partenaires, ce sont quatre-vingts pays qui sont attendus pour le prochain cycle du PISA. Les décideurs du monde entier se servent des résultats de l'enquête PISA aux fins suivantes :

- comparer le niveau de compétence de leurs élèves à celui des élèves des autres pays participants;

- se fixer des objectifs d'amélioration, par exemple atteindre les scores moyens d'autres pays ou parvenir à un degré d'équité plus élevé en termes de perspectives et de résultats éducatifs;

- comprendre les points forts et les points faibles de leur système d'éducation.

Les innombrables rapports rédigés dans les pays participants attestent de l'intérêt que suscite l'enquête PISA au niveau national. Par ailleurs, ses résultats sont abondamment cités dans les débats publics et foisonnent dans les médias du monde entier ${ }^{4}$.

\section{Biblographie}

\section{Sites Internet de référence}

Site PISA de l'OCDE (en anglais) : http://www.pisa.oecd.org/

Site PISA de la DEPP : http://www.educ-eval.education.fr/pisa2003.htm avec accès à tous les exercices libres de diffusion (langue française).

\section{Ouvrages}

BAUTIER E., CRINON J., RAYOU P., ROCHEX J-Y. (2006): «Les performances en littéracie et l'hétérogénéité des univers mentaux mobilisés par les élèves». Revue Cadmo, Université de Rome III.

BAUTIER E. (2005) : «Mobilisation de soi, exigences langagières scolaires et processus de différenciation». Revue Langage et société $n^{\circ} 111$, mars.

BONNET G. (2002): "Reflections in a Critical eye: on the pitfalls of international assessment". Assessment in Education.

BOURNY G., FUMEL S., MONNIER A.-L. et ROCHER T. (2004) : Les élèves de 15 ans - Premiers résultats de l'évaluation internationale PISA 2003. Note d'évaluation de la DEP 04.12, décembre.

BOURNY G., FUMEL S., ROBIN I. et ROCHER T. (2001): Les élèves de 15 ans Premiers résultats d'une évaluation internationale des acquis des élèves. Note d'information 01.52, MJENR-DP\&D, décembre.

BOURNY G., DUPÉ C., RÉMOND M., ROBIN I. et ROCHER T. (2002): "Les compétences des élèves français à l'épreuve d'une évaluation internationale - Premiers résultats de l'enquête PISA $2000 »$. Les Dossiers de la DP\&D n 137, novembre.

4. Voir le site : www.pisa.oecd.org pour des exemples. 
DUPÉ C. et OLIVIER Y. (2005): «Ce que l'évaluation PISA 2003 peut nous apprendre». Le Bulletin vert de l'association des professeurs de mathématiques, $\mathrm{n}^{\circ} 460$, septembreoctobre.

DUPÉ C. et OLIVIER Y. (2002): "L'évaluation PISA». Le Bulletin vert de l'association des professeurs de mathématiques, $\mathrm{n}^{\circ} 439$.

DURU-BELLAT M., MONS N., SUCHAUT B. (2004) : «Inégalités sociales entre élèves et organisation des systèmes éducatifs - quelques enseignements de l'enquête PISA». Les notes de l'IREDU 04/02, mars.

DURU-BELLAT M., MONS N., SUCHAUT B. (2004): "Contextes nationaux, organisation des systèmes éducatifs et inégalités entre élèves : l'éclairage de l'enquête PISA". Politiques d'éducation et de formation $\mathrm{n}^{\circ}$ 9, 2003/3, pp. 95-108.

MEURET D. (2003): Pourquoi les jeunes français ont-ils à 15 ans des compétences inférieures à celles de jeunes d'autres pays? Revue française de pédagogie $\mathrm{n}^{\circ} 142$, janvier-février-mars, pp. 89-104.

MURAT F. et ROCHER T. (2002/2003): «La place du projet professionnel dans les inégalités de réussite scolaire à 15 ans». France Portrait Social, INSEE.

OCDE (1999a): Mesurer les connaissances et compétences des élèves : Un nouveau cadre d'évaluation, OCDE, Paris.

OCDE (2001a): Connaissances et compétences : des atouts pour la vie-Premiers résultats de PISA 2000, OCDE, Paris.

OCDE (2002b): La lecture, moteur de changement: Performances et engagement d'un pays à l'autre - Résultats de PISA 2000, OCDE, Paris.

OCDE (2003e): Cadre d'évaluation de PISA 2003 : connaissances et compétences en mathématiques, lecture, science et résolution de problèmes, OCDE, Paris.

OCDE (2004): Apprendre aujourd'hui, réussir demain - Premiers résultats de PISA 2003.

ROBIN I. et ROCHER T. (2002): «La compétence en lecture des jeunes de 15 ans : une comparaison internationale». Données Sociales, INSEE.

ROBIN I. (2002): "L'enquête PISA sur les compétences en lecture des élèves de 15 ans : trois biais culturels en question». Ville-École-Intégration-Enjeux, $\mathrm{n}^{\circ} 129$, CNDP juin.

ROCHER T. (2003): «La méthodologie des évaluations internationales de compétences». Psychologie et Psychométrie, vol. 24, n² 2/3, pp. 117-146.

REMOND M. (2005) : "Regards croisés sur les évaluations institutionnelles», Repères $\mathrm{n}^{\circ} 31$, pp. 113-140. 